\title{
Brassinosteroid insensitive 1-associated kinase 1 (OsI-BAK1) is associated with grain filling and leaf development in rice
}

\begin{abstract}
Brassinosteroid Insensitive 1 (BRI1)-Associated Kinase I (BAK1) has been reported to interact with BRI1 for brassinosteroid (BR) perception and signal transduction that regulate plant growth and development. The aim of this study is to investigate the functions of a rice OsBAK1 homologue, designated as OsI-BAK1, which is highly expressed after heading. Silencing of OsI-BAK1 in rice plants produced a high number of undeveloped green and unfilled grains compared to the untransformed plants. Histological analyses demonstrated that embryos were either absent or retarded in their development in these unfilled rice grains of OsI-BAK1 RNAi plants. Down regulation of OsI-BAK1 caused a reduction in cell number and enlargement in leaf bulliform cells. Furthermore, transgenic rice plants overexpressing OsI-BAK1 were demonstrated to have corrugated and twisted leaves probably due to increased cell number that caused abnormal bulliform cell structure which were enlarged and plugged deep into leaf epidermis. The current findings suggest that OsI-BAK1 may play an important role in the developmental processes of rice grain filling and leaf cell including the bulliform cells.
\end{abstract}

Keyword: Brassinosteroid; Grains; Overexpression; RNAi; Rice 